\title{
Association of the aspartic acid-repeat polymorphism in the asporin gene with age at onset of knee osteoarthritis in Han Chinese Population
}

\author{
Dongquan Shi · Takahiro Nakamura $\cdot$ Jin Dai $\cdot$ Long Yi \\ Jianghui Qin · Dongyang Chen · Zhihong Xu • Yong Wang · \\ Shiro Ikegawa $\cdot$ Qing Jiang
}

Received: 11 April 2007/Accepted: 20 May 2007/Published online: 30 June 2007

(C) The Japan Society of Human Genetics and Springer 2007

\begin{abstract}
Recent genetic studies for osteoarthritis (OA) have been focused on $A S P N$, the gene encoding asporin, where aspartic acid (D)-repeat polymorphisms are associated with OA in several ethnic groups. The purpose of the present study is to further examine the association of the Drepeat polymorphism in a Han Chinese population. The Drepeat polymorphism was genotyped in 354 knee OA patients ( 257 women and 97 men) who suffered from primary symptomatic knee OA with radiographic confirmation, and the association of the repeat with various clinical parameters was examined. The age at onset (years) in patients with the D14 allele (mean: 51.9, SD: 8.5) was younger than that those without the D14 allele (mean: 54.9, SD: 10.9) $(P=0.023)$. Survival analysis showed D14 was significantly associated with age at onset of OA $(P=0.004$ in the dominant model). The average age at onset of patients with
\end{abstract}

D. Shi · J. Dai · J. Qin · D. Chen · Z. Xu · Q. Jiang $(\bowtie)$ The Center of Diagnosis and Treatment for Joint Disease, Drum Tower Hospital,

affiliated to Medical School of Nanjing University,

Zhongshan Road 321, Nanjing 210008, Jiangsu, China

e-mail: qingj@nju.edu.cn

D. Shi

e-mail:Tim801215@hotmail.com

L. Yi · Y. Wang

Department of Pathology,

Medical School of Nanjing University,

Nanjing, China

S. Ikegawa

Laboratory for Bone and Joint Diseases, Riken, Japan

T. Nakamura

Laboratory for Statistical Analysis, SNP Research Center,

Riken, Japan the D13/D13 genotype (mean: 56.1, SD: 11.1) was older than those without the D13/D13 genotype (mean: 53.0, SD: 9.9) $(P=0.013)$. Our study further highlighted the significance of asporin in OA.

Keywords Asporin - Association - Knee osteoarthritis · Age at onset - Han Chinese

\section{Introduction}

Osteoarthritis (OA, OMIM 165720) is the most prevalent form of arthritis. A recent study in China show that the prevalence of radiographic knee OA increased in proportion to age: $13.5,41.9,64.1$, and $71.4 \%$ for the $40-, 50-, 60-$, and 70-year-old age groups, respectively (Du et al. 2005). The risk of disability in mobility in knee OA is greater than any other diseases in the people age $\geq 65$ years (Hunter et al. 2006). Genetic factors have been implicated in its etiology (Ikegawa et al. 2003; Loughlin 2005) and several susceptibility genes with reasonable functional significance have been reported (Loughlin et al. 2004; Kizawa et al. 2005; Mototani et al. 2005; Miyamoto et al. 2007).

Recent genetic studies for OA have been focused on $A S P N$, the gene encoding asporin (Kizawa et al. 2005; Mustafa et al. 2005; Kaliakatsos et al. 2006; RodriguezLopez et al. 2006; Ikegawa et al. 2006; Jiang et al. 2006). Asporin is a new member of the small leucin-rich proteoglycan family (Lorenzo et al. 2001; Henry et al. 2001). Asporin has consecutive aspartic-acid residues (D-repeat) in the amino-terminal region of its mature protein encoded by a microsatellite polymorphism. Kizawa et al. (2005) first reported a strong association of $A S P N$ with knee and hip OA in Japanese. Together with convincing functional evidence, they show that the D14 allele (an allele 
containing 14 D-repeats) is over-represented, and the D13 allele is under-represented in OA. The replication of association was not clear in the subsequent studies in European Caucasians (Mustafa et al. 2005; Kaliakatsos et al. 2006; Rodriguez-Lopez et al. 2006), but was definitely positive in the Han Chinese population (Jiang et al. 2006).

The purpose of the present study is to further examine the association of the D-repeat polymorphism in asporin for various clinical parameters in the Han Chinese population. We found the significant association of D-repeat with age at onset of OA, which has not been reported in the previous studies.

\section{Patients and methods}

Subjects, DNA samples and genotyping

A total of 354 knee OA patients (257 women and 97 men) were enrolled consecutively at Center of Diagnosis and Treatment for Joint Disease, Drum Tower Hospital, affiliated to Medical School of Nanjing University. All subjects included in the study were Han Chinese who are living in and around Nanjing. The inclusion and exclusion criteria were as described previously (Jiang et al. 2006). Clinical information including age, sex, body mass index (BMI), and age at onset were collected by participating doctors from the subjects at their visits to the hospital. Age at onset is defined as the year when patients began to have not only definite signs and symptoms of OA, but also radiographic evidence. No subjects dropped out during the process of the study. The study protocol was approved by an ethical committee of Medical School of Nanjing University. A written informed consent was obtained from patients. Extraction of genomic DNA and genotyping of the ASPN D-repeat polymorphisms were as described previously (Jiang et al. 2006).

\section{Statistical analysis}

Association of the ASPN D-repeat polymorphisms was tested for asporin genotypes by stratification with a variety of clinical information. $\chi^{2}$ test was used to assess the
Hardy-Weinberg equilibrium. Mann-Whitney and KruskalWallis tests were used in this study. We also applied survival analysis using Kaplan-Meier method and log-rank test for the data.

\section{Results}

The age of the knee OA patients (mean \pm SD) was $60.6 \pm 10.9$ (range 40-89) years and the age at onset $($ mean \pm SD) was $54.4 \pm 10.6$ (range 18-77) BMI of the patients was $26.6 \pm 6.8 \mathrm{~kg} / \mathrm{m}^{2}$. Over $60 \%$ of patients had a $\mathrm{K} / \mathrm{L}$ score of three or four. Seven different alleles containing from 11 to 17 D-repeats were identified, which composed 17 genotypes. Frequencies of alleles were shown in Table 1. Frequencies of genotypes were similar to the previous study (Jiang et al. 2006). Distributions of genotypes were conformed to Hardy-Weinberg equilibrium $(P=0.98)$.

To evaluate the effect of $A S P N$ for the age at onset of knee OA, we examined the difference of the age at onset of knee OA between the genotypes using the Mann-Whitney and Kruskal-Wallis tests. The age at onset in patients with the D14 allele (mean: 51.9 years, SD: 8.5) was younger than that those without the D14 allele (mean: 54.9 years, SD: 10.9) $(P=0.023)$. We also applied survival analysis using the Kaplan-Meier method and the log-rank test for the date (Table 2 and Fig. 1). The log-rank test showed significant difference between the D-repeat genotypes (in the dominant mode: $P=0.004$, in the genotype mode: $P=0.016)$. When stratified by gender, similar results were found in female patients (dominant mode: $P=0.007$, genotype mode: $P=0.026$ ). The age at onset years in female patients with the D14 allele (mean: 51.8, SD: 7.7) was younger than that those without the D14 allele (mean: 54.8, SD: 10.6). No significant results were detected in male samples $(P>0.05)$ and in stratification by age, $\mathrm{K} / \mathrm{L}$ grade, and BMI in all subjects (all $P>0.05$ ). When we focused on survival functions of patients with and without D13/D13 genotype, significant difference of onset age was detected by log-rank test $(P=0.001)$. The average age at onset of patients with D13/D13 genotype (mean: 56.1, SD: 11.1) was older than those without D13/D13 genotype (mean: 53.0, SD: 9.9), and a statistically significant difference
Table 1 Allelic frequency of the aspartic acid (D)-repeat polymorphism of asporin in knee osteoarthritis (KOA) in a Han Chinese population

\begin{tabular}{llllllllll}
\hline Group & $\begin{array}{l}\text { No. of } \\
\text { subject }\end{array}$ & \multicolumn{2}{l}{ No. of allele (\%) } \\
\cline { 3 - 10 } & & D11 & D12 & D13 & D14 & D15 & D16 & D17 & Total \\
\hline All & 354 & $1(0.1)$ & $116(16.4)$ & $477(67.3)$ & $62(8.8)$ & $16(2.3)$ & $28(4.0)$ & $8(1.1)$ & 708 \\
Female & 257 & $1(0.2)$ & $87(16.9)$ & $341(66.4)$ & $46(8.9)$ & $12(2.3)$ & $21(4.1)$ & $6(1.2)$ & 514 \\
Male & 97 & 0 & $29(14.9)$ & $136(70.1)$ & $16(8.3)$ & $4(2.1)$ & $7(3.6)$ & $2(1.0)$ & 194 \\
\hline
\end{tabular}


Table 2 The results of analysis for the age at onset

\begin{tabular}{|c|c|c|c|c|c|c|c|c|c|}
\hline \multirow[t]{2}{*}{ Allele } & \multirow[t]{2}{*}{ Mode of inheritance } & \multicolumn{2}{|c|}{ Allele $1^{\mathrm{a}}$} & \multicolumn{2}{|l|}{ Both $^{\mathrm{a}}$} & \multicolumn{2}{|c|}{ Allele $2^{\mathrm{a}}$} & \multicolumn{2}{|l|}{$P$-value } \\
\hline & & Mean & SD & Mean & SD & Mean & SD & Non-parametric test & Log-rank test \\
\hline D13 & Dominant & 54.8 & 10.8 & - & - & 51.7 & 8.4 & 0.105 & 0.018 \\
\hline D13 & Recessive & 56.1 & 11.1 & - & - & 53.0 & 9.9 & 0.013 & 0.001 \\
\hline D13 & Genotype & 56.1 & 11.1 & 52.3 & 10.2 & 51.7 & 8.4 & 0.031 & 0.001 \\
\hline D14 & Dominant & 51.9 & 8.5 & - & - & 54.9 & 10.9 & 0.023 & 0.004 \\
\hline D14 & Recessive & 53.5 & 2.1 & - & - & 54.4 & 10.6 & 0.959 & 0.593 \\
\hline D14 & Genotype & 53.5 & 2.1 & 51.9 & 8.6 & 54.9 & 10.9 & 0.072 & 0.016 \\
\hline D12 & Dominant & 52.6 & 10.5 & - & - & 55.2 & 10.5 & 0.085 & 0.040 \\
\hline
\end{tabular}

${ }^{a}$ If the mode of inheritance is dominant, allele 1 means genotype with target allele and allele 2 means genotype without target allele. If the mode of inheritance is recessive, allele 1 means homozygote of target allele and allele 2 means genotypes except for homozygote of target allele. If the mode of inheritance is genotype, allele 1, both, allele 2 mean homozygote of target allele, heterozygote of target allele, genotype without target allele, respectively

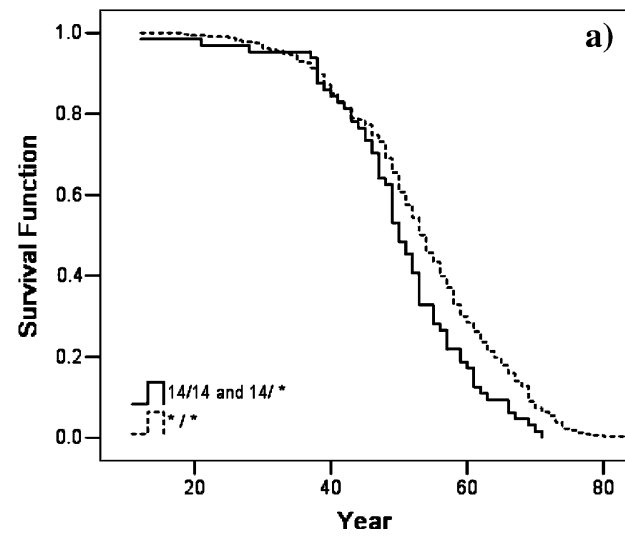

Fig. 1 Survival functions of patients with the D14 allele and without the D14 allele (a), and of those with D13/D13 genotype and without D13/D13 genotype (b). a Average onset age of patients with D14 allele $(n=60)$ was significantly earlier $(P=0.004)$ than without D14

was found $(P=0.013)$. No significant differences were detected when we focused on other alleles of ASPN (all $P>0.05$, shown in the Table 2).

\section{Discussion}

Not only susceptibility to disease occurrence of OA, but also various aspects of $\mathrm{OA}$, including its progression, severity, and response to certain treatment can be associated with genetic determinant(s). In the present study, we found that the D-repeat polymorphism in ASPN is associated with the onset of knee OA in Han Chinese population. Patients with D14 allele, the susceptibility allele had earlier onset, which is consistent with its suspected pro-OA function in our previous studies (Kizawa et al. 2005; Nakajima et al. manuscript in submission). Our data also suggest the protective effect of D13 allele for onset of OA.

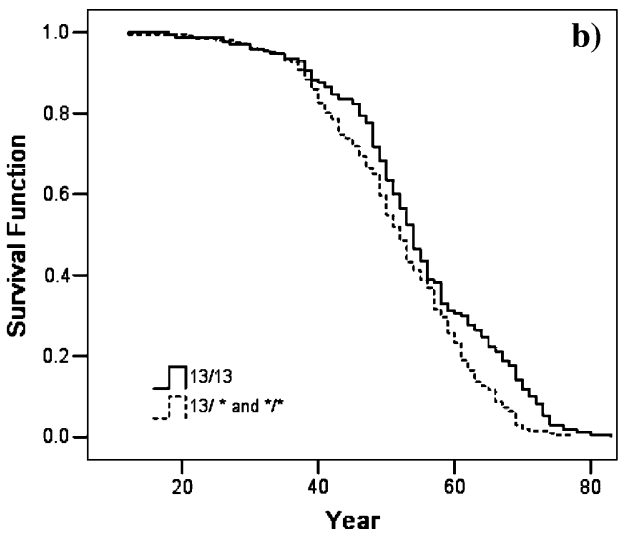

patients $(n=294)$. b Average onset age of patients with D13/D13 genotype $(n=160)$ was earlier $(P=0.001)$ than patients without D13/D13 genotype $(n=194)$

However, functional study does not support it, as the inhibitory effect of D13 allele on TGF- $\beta$ function is not lower than other alleles except for the D14 allele. Later onset of OA in D13 homozygotes may be just the reflection of their not having the D14 allele. Valdes et al. (2007) detected several important candidate gene associations with OA and the fact that many of these associations were strongly dependent on sex and ethnicity and were often probably site specific. We also detected different results in stratification by sex when age at onset in patients with or without the D14 allele was compared. Just as in the susceptibility to the disease, the present result also needs replication in other populations, preferably of different ethnic origins. Well-designed, prospective studies will answer the question.

$A S P N$ is the second gene that associated with age at onset of knee OA. Williams et al. (1993) found that the type II pro-collagen gene (COL2A1) is associated with 
severe, early-onset knee OA. We found no association of $A S P N$ with severity of OA, neither in stratification by age, $\mathrm{K} / \mathrm{L}$ grade, BMI. $A S P N$ is also reported to be associated with clinical outcome of rheumatoid arthritis (Torres et al. 2007). Individuals with D14 produced rheumatoid factor more often than the rest. The age of onset is higher in the group of individuals harboring D13 allele compared with the rest $(P=0.001)$. TGF- $\beta$ is a key growth factor for bone and joint metabolism. The supposed ASPN function to the pathogenesis of OA, excessive inhibition of TGF- $\beta$ can be applicable to other bone and joint diseases. ASPN may influence outcome of a broad spectrum of bone and joint diseases.

Acknowledgments This work is supported by National Nature Science Foundation of China (30571874) and Programme of Technology Development of Nanjing (200603001).

\section{References}

Du H, Chen SL, Bao CD, Wang XD, Lu Y, Gu YY, Xu JR, Chai WM, Chen J, Nakamura H, Nishioka K (2005) Prevalence and risk factors of knee osteoarthritis in Huang-Pu district, Shanghai, China. Rheumatol Int 25(8):585-590

Hunter DJ, Felson DT (2006) Osteoarthritis. BMJ 332(7545):639-642

Ikegawa S, Ikeda T, Mabuchi A (2003) Genetic analysis of osteoarthritis: toward identification of its susceptibility genes. J Orthop Sci 8(5):737-739

Jiang Q, Shi D, Yi L, Ikegawa S, Wang Y, Nakamura T, Qiao D, Liu C, Dai J (2006) Replication of the association of the aspartic acid repeat polymorphism in the asporin gene with knee-osteoarthritis susceptibility in Han Chinese. J Hum Genet 51(12):1068-1072

Kaliakatsos M, Tzetis M, Kanavakis E, Fytili P, Chouliaras G, Karachalios T, Malizos K, Tsezou A (2006) Asporin and knee osteoarthritis in patients of Greek origin. Osteoarthritis Cartilage 14(6):609-611

Kellgren JH, Lawrence JS (1963) Radiological assessment of osteoarthrosis. Ann Rheum Dis 22:237-255

Kizawa H, Kou I, Iida A, Sudo A, Miyamoto Y, Fukuda A, Mabuchi A, Kotani A, Kawakami A, Yamamoto S, U chida A, Nakamura K, Notoya K, Nakamura Y, Ikegawa S (2005) An aspartic acid repeat polymorphism in asporin inhibits chondrogenesis and increases susceptibility to osteoarthritis. Nat Genet 37(2):138-144

Lorenzo P, Aspberg A, Onnerfjord P, Bayliss MT, Neame PJ, Heinegard D (2001) Identification and characterization of asporin. a novel member of the leucine-rich repeat protein family closely related to decorin and biglycan. J Biol Chem 276:12201-12211

Loughlin (2005) The genetic epidemiology of human primary osteoarthritis: current status. Expert Rev Mol Med 7:1-12

Loughlin J, Dowling B, Chapman K, Marcelline L, Mustafa Z, Southam L, Ferreira A, Ciesielski C, Carson DA, Corr M (2004) Functional variants within the secreted frizzled-related protein 3 gene are associated with hip osteoarthritis in females. Proc Natl Acad Sci USA 101(26):9757-9762

Miyamoto Y, Mabuchi A, Shi D, Kubo T, Takatori Y, Saito S, Fujioka M, Sudo A, Uchida A, Yamamoto S, Ozaki K, Takigawa M, Tanaka T, Nakamura Y, Jiang Q, Ikegawa S (2007) A functional polymorphism in the 5' UTR of GDF5 is associated with susceptibility to osteoarthritis. Nat Genet 39(4):529-533

Mototani H, Mabuchi A, Saito S, Fujioka M, Iida A, Takatori Y, Kotani A, Kubo T, Nakamura K, Sekine A, Murakami Y, Tsunoda T, Notoya K, Nakamura Y, Ikegawa S (2005) A functional single nucleotide polymorphism in the core promoter region of CALM1 is associated with hip osteoarthritis in Japanese. Hum Mol Genet 14(8):1009-1017

Mustafa Z, Dowling B, Chapman K, Sinsheimer JS, Carr A, Loughlin $\mathrm{J}$ (2005) Investigating the aspartic acid (D) repeat of asporin as a risk factor for osteoarthritis in a UK Caucasian population. Arthritis Rheum 52(11):3502-3506

Rodriguez-Lopez J, Pombo-Suarez M, Liz M, Gomez-Reino JJ, Gonzalez A (2006) Lack of association of a variable number of aspartic acid residues in the asporin gene with osteoarthritis susceptibility: case-control studies in Spanish Caucasians. Arthritis Res Ther 8(3):R55

Torres B, Orozco G, Garcia-Lozano JR, Oliver J, Fernandez O, Gonzalez-Gay MA, Balsa A, Garcia A, Pascual-Salcedo D, Lopez-Nevot MA, Nunez-Roldan A, Martin J, Gonzalez-Escribano MF (2007) Asporin repeat polymorphism in rheumatoid arthritis. Ann Rheum Dis 66(1):118-120

Valdes AM, Loughlin J, Oene MV, Chapman K, Surdulescu GL, Doherty M, Spector TD (2007) Sex and ethnic differences in the association of ASPN, CALM1, COL2A1, COMP, and FRZB with genetic susceptibility to osteoarthritis of the knee. Arthritis Rheum 56(1):137-146

Walsh PS, Metzger DA, Higuchi R (1991) Chelex 100 as a medium for simple extraction of DNA for PCR-based typing from forensic material. Biotechniques 10:506-513

Williams CJ, Considine EL, Knowlton RG, Reginato A, Neumann G, Harrison D, Buxton P, Jimenez S, Prockop DJ (1993) Spondyloepiphyseal dysplasia and precocious osteoarthritis in a family with an Arg75->Cys mutation in the procollagen type II gene (COL2A1). Hum Genet 92(5):499-505 